\title{
Intermolecular 1,3-dipolar cycloadditions of azomethine imines
}

\author{
Raymond C. F. Jones, ${ }^{*, a}$ Stephen J. Hollis, ${ }^{\text {b }}$ and James N. Iley*,b \\ ${ }^{a}$ Department of Chemistry, Loughborough University, Leics. LE11 3TU, UK \\ ${ }^{b}$ Department of Chemistry, The Open University, Walton Hall, Milton Keynes MK7 6AA, UK \\ E-mail: r.c.f.jones@lboro.ac.uk
}

\section{Dedicated to Professor Lutz Tietze to mark his $65^{\text {th }}$ birthday}

\begin{abstract}
Dipolar cycloadditions of azomethine imines, formed in situ from aldehydes and $N^{1}$-alkyl- $N^{2}$ acylhydrazines, with electron-deficient dipolarophiles produce pyrazolidines: mono-substituted dipolarophiles afford principally 4-substituted pyrazolidines
\end{abstract}

Keywords: 1,3-dipole, azomethine imine, pyrazolidine

\section{Introduction}

As part of our research effort into the preparation of peptidomimetics containing 5-membered heterocyclic rings, ${ }^{1}$ we wished to investigate the synthesis of pyrazolidines (tetrahydropyrazoles) 1, Scheme 1.

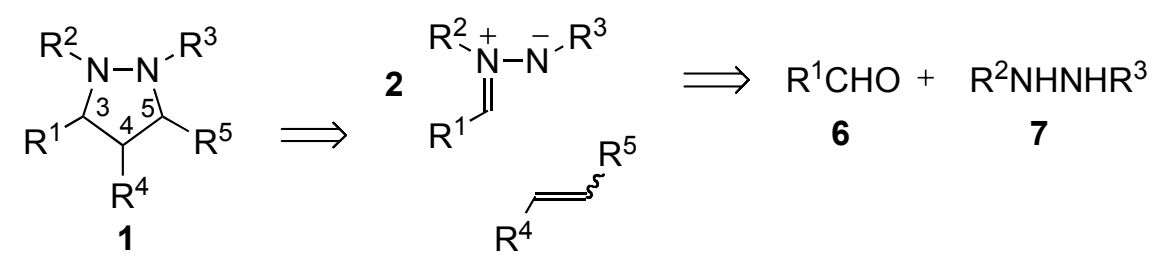

Scheme 1. Retrosynthetic analysis of pyrazolidines.

Our ultimate targets were constrained mimics of the types $\mathbf{3}$ and $\mathbf{4}$ as replacements for the dipeptide unit $\mathbf{5}^{2}$ The incorporation of a cyclic moiety into a peptide backbone can restrict the conformational freedom of the peptide and these so-called peptidomimetics ${ }^{3}$ can possess enhanced biological activity or increased selectivity towards specific receptor sites. 
<smiles>[R]C(N)C1CC(C(=O)O)NN1</smiles>

3<smiles>[R]C(N)C1NNCC1C(=O)O</smiles>

4<smiles>[R]C(N)C(=O)NC([R])C(=O)O</smiles>

5

The pyrazolidine ring is accessible via 1,3-dipolar cycloaddition reaction of an azomethine imine 2 with an appropriately substituted alkene (Scheme 1), that has the potential to generate three new chiral centers at C-3, C-4 and C-5. It is perhaps not surprising that many of the reported examples of cycloadditions involving azomethine imines have been intramolecular, allowing some control to be exercised over the regio- and stereochemistry of the product. $^{5}$ Azomethine imines are available from the addition to an aldehyde, $\mathbf{6}$, of a 1,2-disubstituted hydrazine 7, ${ }^{6}$ resulting in the formation of an aminol, 8 (Scheme 2) that loses the elements of water to form the dipole. ${ }^{7}$ Certain restrictions are placed on the hydrazine substituents; if $\mathrm{R}^{2}=\mathrm{H}$, then the aminol eliminates water to form a stable hydrazone, and it is also usual for $\mathrm{R}^{3}$ to be an electron-withdrawing group in order to stabilize the formal negative charge on the dipole $\mathbf{2}$.

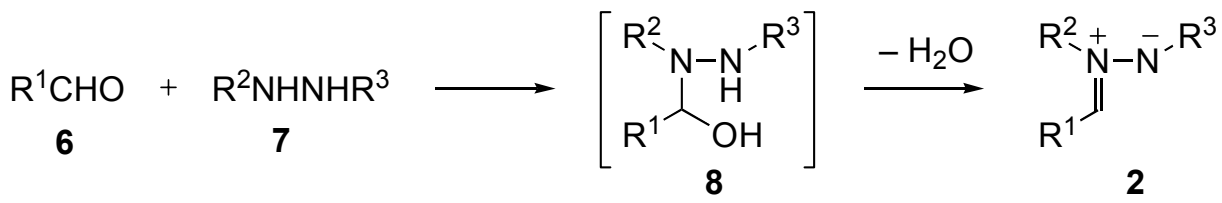

Scheme 2. Formation of azomethine imines.

In order to progress towards our long-term objective, and since intermolecular cycloadditions with azomethine imines had been relatively little studied, we have examined the scope and limitations of the reaction, investigating the effects of varying the nature of the different components: the aldehyde, hydrazine and dipolarophile. The results of this exploratory work are described herein: our report is prompted by recent related studies using rigid cyclic azomethine ylides. ${ }^{8,9}$

\section{Results and Discussion}

As an orienting cycloaddition, benzaldehyde (lacking $\alpha$-hydrogen atoms), $N^{1}$-acetyl- $N^{2}$ methylhydrazine 9 (selected as meeting the criteria outlined earlier, and prepared simply from ethyl acetate and methylhydrazine ${ }^{10}$ ) and methyl propenoate (a simple mono-substituted dipolarophile) were reacted together (Scheme 3) in toluene at reflux for $72 \mathrm{~h}$ under Dean-Stark water removal. ${ }^{11}$ 


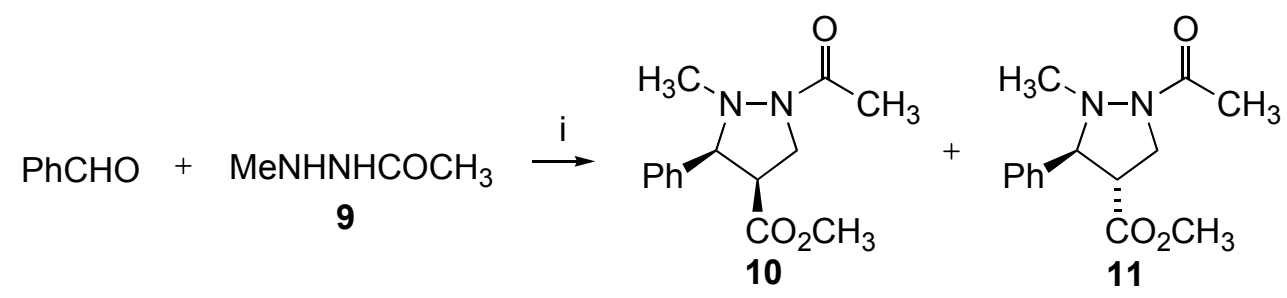

Scheme 3. Reagent: i, $\mathrm{CH}_{2}=\mathrm{CHCO}_{2} \mathrm{Me}, \mathrm{PhMe}$ reflux, Dean-Stark trap.

Chromatographic purification of the crude product afforded both the syn- (10) and the anti(11) 4-methoxycarbonylpyrazolidine diastereoisomers (17 and 22\%, respectively). The regiochemistry of the products was elucidated by $2 \mathrm{D}-\mathrm{NMR}$ spectroscopy, the ${ }^{1} \mathrm{H}-{ }^{1} \mathrm{H}$ COSY spectrum revealing coupling between the methine protons, showing them to be adjacent ( $\mathrm{C}-3$ and $\mathrm{C}-4)$. The relative stereochemistry of the anti isomer $\mathbf{1 1}$ was deduced by n.O.e. experiments and confirmed by an X-ray crystal structure determination (Fig. 1).

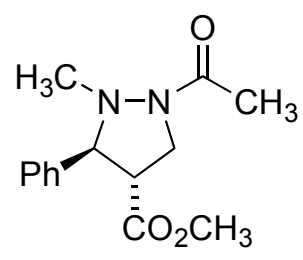

11

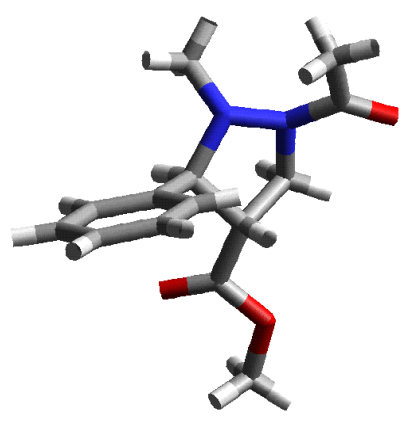

Figure 1. X-Ray crystal structure of anti-tetrahydropyrazolidine 11.

Whereas the syn-isomer $\mathbf{1 0}$ was obtained pure following column chromatography, preparative HPLC was necessary to separate the anti-isomer $\mathbf{1 1}$ from a trace impurity. Although it was not possible to isolate this latter material in pure form, it was tentatively assigned as the 5methoxycarbonylpyrazolidine $\mathbf{1 2}$ based on its signals in the ${ }^{1} \mathrm{H}$ NMR spectrum of the mixture. Also isolated from the reaction mixture were the trisubstituted hydrazine 13 (33\%; incompletely characterized), formed by conjugate addition of the hydrazine to methyl propenoate, ${ }^{8}$ and the hexahydrotetrazine 14 (27\%) presumably arising from dimerization of the dipole, a reaction that has been observed by others. ${ }^{6,7,12}$ In an attempt to improve the efficiency of the reaction and minimize the formation of these unwanted by-products, the effect of altering the reaction conditions was investigated. 


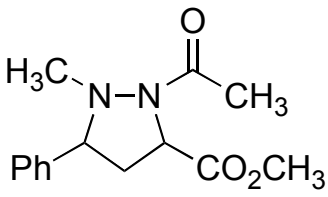

12

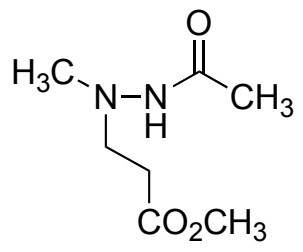

13<smiles>CC(=O)N1C(=O)N(C)C(c2ccccc2)N(C)C1c1ccccc1</smiles>

14

Although the use of a Dean-Stark trap would appear necessary to promote formation of the dipole, cycloadditions of azomethine imines have been carried out in the presence of a molar equivalent of water. ${ }^{8}$ When the reaction was attempted either without water removal or with one molar equivalent of water added, a reduction in yield of the cycloadducts $\mathbf{1 0}$ and $\mathbf{1 1}$ and the hexahydrotetrazine 14 was observed, suggesting that dipole formation had been inhibited. A significant proportion of the azomethine imine dimerizes to form 14 in preference to cycloaddition. Triethylamine has been reported as an effective suppressant of dipole dimerization during the cycloaddition of azomethine imines; ${ }^{13}$ however, a reaction performed in the presence of 3 molar equivalents of base did not result in a significant drop in the quantity of dimer $\mathbf{1 4}$ formed. Lowering the reaction temperature to that of benzene at reflux, or to ambient temperature (using $4 \AA$ molecular sieves to remove water), gave reduced yields of $\mathbf{1 0}$ and $\mathbf{1 1}$. To minimize the conjugate addition (to form 13) that competes with dipole formation, an extra molar equivalent of aldehyde was used but this resulted in only a slight increase in the yields of 10 and 11. Increasing the amount of hydrazine present so that complete conversion of the aldehyde to the dipole could take place despite any conjugate addition likewise produced a similar, marginal improvement. We were thus unable to improve significantly upon the initial yield, and so turned our attention towards the reaction of the azomethine imine with other dipolarophiles (Scheme 4).

Cycloaddition of the azomethine imine with acrylonitrile gave the syn- and anti-4cyanotetrahydropyrazoles $15(15 \%)$ and $16(10 \%)$ respectively. The regiochemistry of the products was again determined by 2D NMR spectroscopy. The relative stereochemistry was assigned by comparison with the ${ }^{1} \mathrm{H}$ NMR spectra of the 4-methoxycarbonyltetrahydropyrazoles 10 and 11, for which $\mathrm{C}-4(\mathrm{H})$ resonates at $\delta 3.94$ for the $s y n$-isomer $\mathbf{1 0}$ and $\delta 3.45$ for $\mathbf{1 1}$. The corresponding values for cycloadducts $\mathbf{1 5}$ and $\mathbf{1 6}$ are $\delta 3.78$ and $\delta 3.44$, respectively. In addition, the coupling constant ${ }^{3} J_{3,4}$ is larger in isomer 15 than in $\mathbf{1 6}(6.6 \mathrm{~Hz}$ compared to $6.0 \mathrm{~Hz})$ which compares favorably with the values for 10 and $11(7.7 \mathrm{~Hz}$ and $5.9 \mathrm{~Hz}$, respectively). The antiisomer 16 was not isolated in pure form as it contained traces of an impurity (assigned from the ${ }^{1} \mathrm{H}$ NMR spectrum of the mixture as the 5-substituted pyrazolidine) inseparable by chromatography.

When the symmetrical 1,2-disubstituted dipolarophile, dimethyl fumarate was used, the anti, anti-product 17 (38\%; stereochemistry determined by n.O.e. experiments) was isolated along with an inseparable mixture of further $17(21 \%)$ and an isomer tentatively assigned from the ${ }^{1} \mathrm{H}$ NMR spectrum as the syn, anti-product $18(6 \%)$. Dimethyl maleate as dipolarophile afforded 
only one pure isolated product (11\%), all-syn 19, assigned on the basis of n.O.e. experiments. A mixture of further unidentified cycloaddition products $(22 \%)$ was also obtained. Only two diastereoisomers, the all-syn 19 and the anti, syn-isomer (having both ester substituents anti- to the phenyl substituent) can arise from concerted cycloaddition. However, since at least three different cycloadducts appeared to be formed, this suggests some conversion of dimethyl maleate into the more stable fumarate before cycloaddition. Reaction of the dipole with $\mathrm{N}$ phenylmaleimide, usually a very reactive dipolarophile, resulted in isolation of one cycloadduct, the anti, syn-isomer $\mathbf{2 0}$ (stereochemistry again determined by n.O.e. studies) in a disappointing yield of $4 \%$.

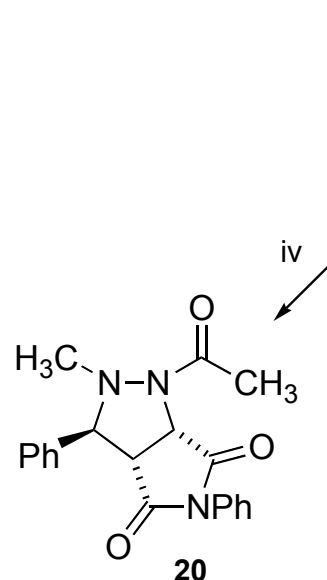

20

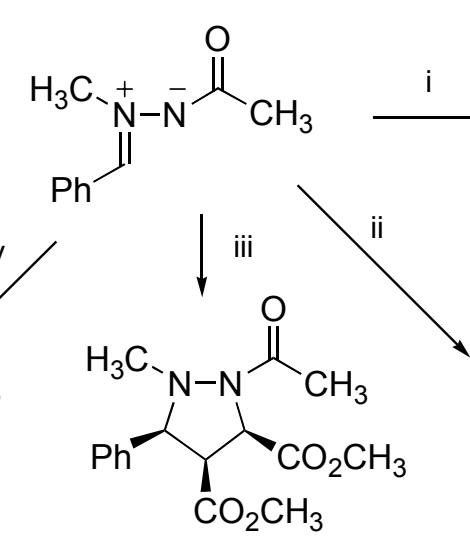

19<smiles>CC(=O)N1C[C@H](C#N)[C@H](P)N1C</smiles><smiles>COC(=O)[C@H]1[C@@H](C(C)=O)N(C(C)=O)N1C</smiles>

17<smiles>CC(=O)N1C[C@H](C#N)[C@H](P)N1C</smiles><smiles>COC(=O)[C@H]1[C@@H](C(C)=O)N(C(C)=O)N1C</smiles>

18

Scheme 4. Reagents: i, $\mathrm{CH}_{2}=\mathrm{CHCN}$; ii, $\quad E-\mathrm{MeO}_{2} \mathrm{CCH}=\mathrm{CHCO}_{2} \mathrm{Me}$; iii, Z$\mathrm{MeO}_{2} \mathrm{CCH}=\mathrm{CHCO}_{2} \mathrm{Me}$; iv, $\mathrm{N}$-phenylmaleimide (PhMe reflux, Dean-Stark trap).

We next examined the effect of altering the aldehyde. Replacing benzaldehyde by 2methylpropanal (heating only for $16 \mathrm{~h}$ ) gave the 4-substituted pyrazolidines 21 and 22, in 25\% and $11 \%$ yield, respectively (Scheme 5). The regiochemistry was determined by 2D NMR spectroscopy, the stereochemistry by comparison with data for $\mathbf{1 0}$ and $\mathbf{1 1} .^{14}$ Also isolated was the 5-substituted isomer $\mathbf{2 3}$ in 1\% yield (stereochemistry undetermined).

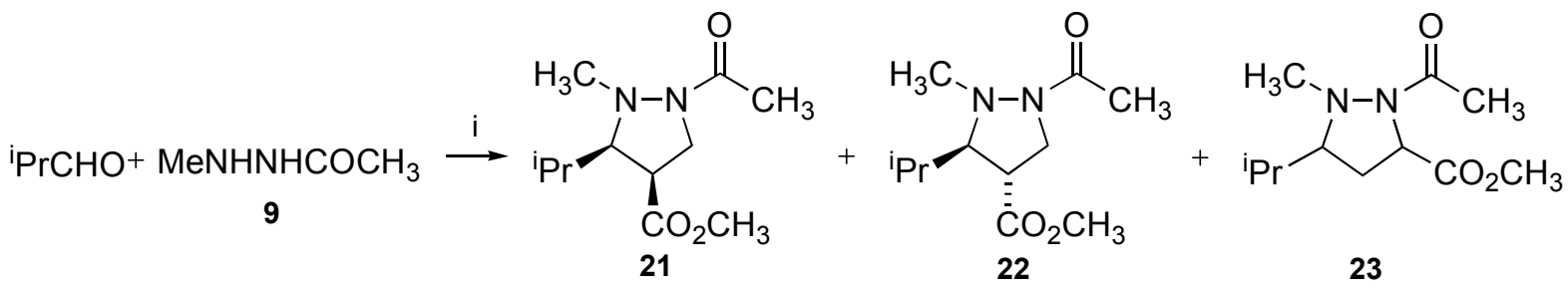

Scheme 5. Reagent (i), $\mathrm{CH}_{2}=\mathrm{CHCO}_{2} \mathrm{Me}$, PhMe reflux, Dean-Stark trap. 
Cycloaddition is thus possible when the aldehyde bears a simple alkyl or aryl group. Next we examined the use of more functionalized aldehydes, particularly those that would offer the exocyclic amino functionality present in the tetrahydropyrazoles 3 and 4. Subjecting the Bocprotected $\alpha$-amino aldehyde $\mathbf{2 4}$ (synthesized from $S$-alanine ${ }^{1 \mathrm{a}, 15}$ ) to the normal cycloaddition conditions with methyl acrylate did not give the expected adducts, but instead gave cyclization involving the carbamate functionality to afford the dihydrooxazole 25 (27\%) (Scheme 6). The $N, N$-dibenzylated aldehyde 26 (prepared by tribenzylation of $S$-alanine with $\mathrm{PhCH}_{2} \mathrm{Br}-\mathrm{K}_{2} \mathrm{CO}_{3}$, reduction of the benzyl ester using $\mathrm{LiAlH}_{4}$, and Swern oxidation of the resulting 2dibenzylaminopropan-1-ol ${ }^{16}$ ) was employed to prevent this, but instead gave rise to the 2,3dihydro-1,3,4-oxadiazole 27 from an alternative cyclization involving the hydrazine $N$-acetyl group (Scheme 6). 2-Benzyloxypropanal 28 (prepared by benzylation of ethyl lactate using $\mathrm{PhCH}_{2} \mathrm{Br}-\mathrm{NaH}$ and reduction of the ester with DIBAL at $-50^{\circ} \mathrm{C}$ ) gave the analogous product 29. Products 25, 27, and 29 were incompletely characterized. The presence of an electronegative atom $\alpha$ - to the dipole carbon atom thus seems to encourage formation of charge-neutral molecules 27 and 29 over reaction with the dipolarophile; this may be because of a destabilization of the dipole. Attempts to carry out cycloadditions using 2-oxopropanal (methyl glyoxal) or ethyl glyoxylate as aldehyde component were unsuccessful, the only product isolated in each case being the conjugate addition product 13 (see above).
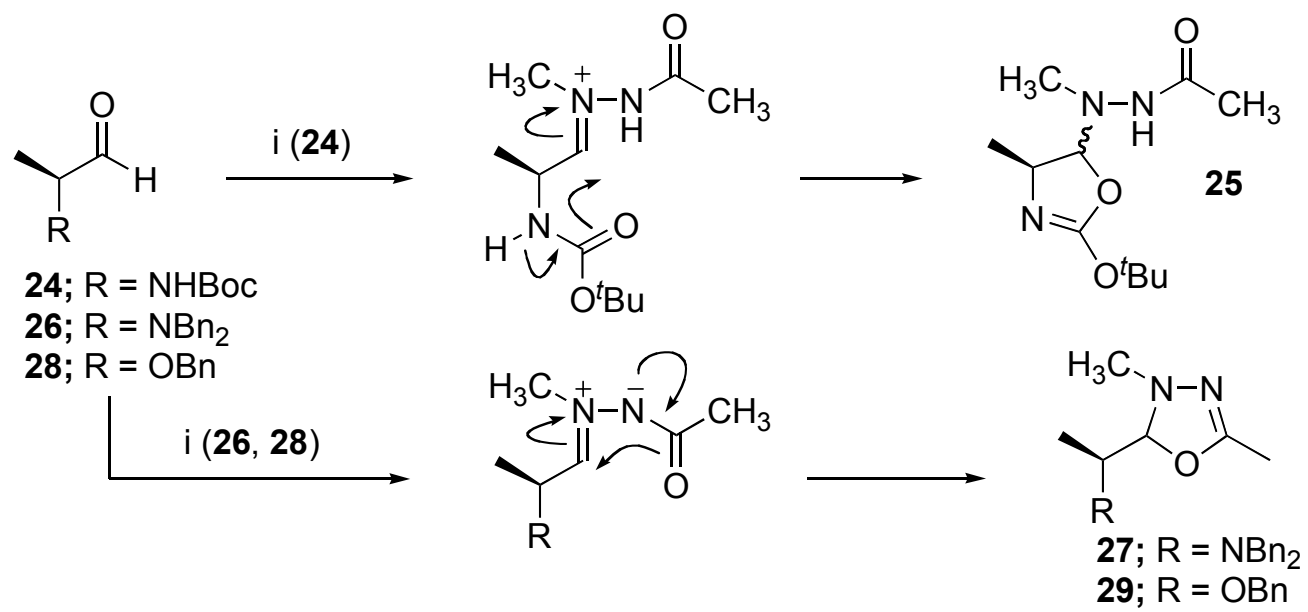

Scheme 6. Reagent: i, $\mathrm{MeNHNHCOCH}_{3}$ (9), PhMe reflux, Dean-Stark trap.

We next turned our attention to two further hydrazines. The $N^{1}$-acetyl- $N^{2}$-benzylhydrazine 30, formed via reductive alkylation of $N$-acetylhydrazine using benzaldehyde, ${ }^{17}$ afforded cycloadduct 31 in only 4\% yield upon reaction with 2-methylpropanal and methyl propenoate (Scheme 7) in a sealed tube for $72 \mathrm{~h}$, with molecular sieves being used to remove water. The increased steric bulk of the hydrazine substituent seemed to hinder the cycloaddition. We assume this adduct to be a 4-methoxycarbonylpyrazolidine 31, based on the precedent above, although it 
proved impossible to determine unequivocally either the regio- or stereo-chemistry of this product as the $\mathrm{C}-4$ and $\mathrm{C}-5$ methine protons were indistinguishable by NMR spectroscopy.

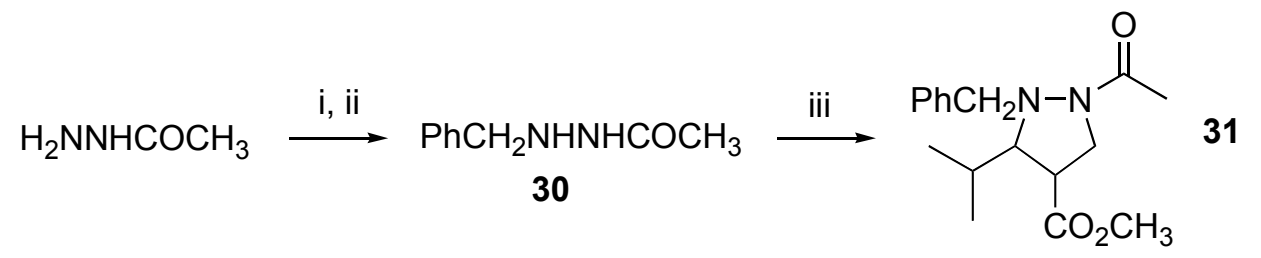

Scheme 7. Reagents: i, PhCHO, PhMe reflux; ii, $\mathrm{NaBH}_{3} \mathrm{CN}, p-\mathrm{TsOH} ; 1 M \mathrm{NaOH}$; iii, $i$-PrCHO, $\mathrm{CH}_{2}=\mathrm{CHCO}_{2} \mathrm{Me}$, molecular sieves, sealed tube.

Similarly, $N^{1}$-tert-butoxycarbonyl- $N^{2}$-methylhydrazine $32^{18}$ afforded the tetrahydropyrazole $33(18 \%)$ as the only cycloadduct from reaction with benzaldehyde and methyl propenoate (Scheme 8). Once again the structure of this product is assumed to be that shown, although the signals for $\mathrm{C}-4(\mathrm{H})$ and one of the methylene protons could not be distinguished by NMR spectroscopy, hindering elucidation of the regio- and stereochemistry.

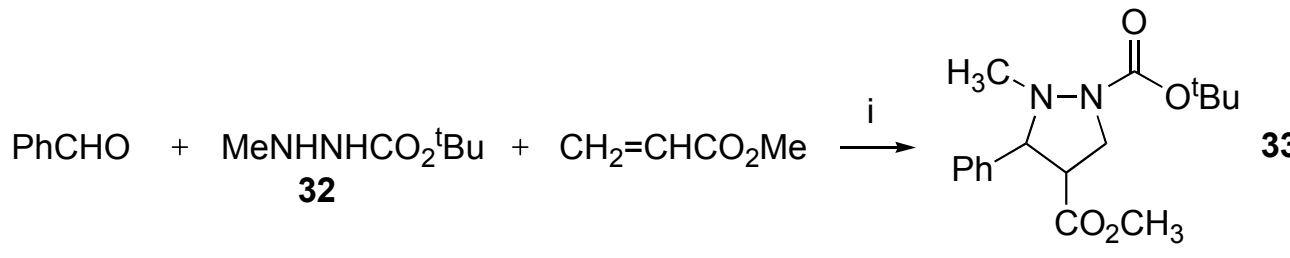

Scheme 8. Reagents: i, PhMe, reflux.

\section{Conclusions}

The 1,3-dipolar cycloadditions of azomethine imine dipoles that have been described demonstrate that the reaction proceeds with electron-deficient alkene dipolarophiles to provide pyrazolidines; mono-substituted dipolarophiles afford predominantly 4-substituted pyrazolidines. ${ }^{6-8}$ A variety of mono- and 1,2-disubstituted dipolarophiles can be used, but the cycloaddition is more sensitive to structural variations in the hydrazine and aldehyde. No cycloadditions were observed with an aldehyde bearing an $\alpha$-heteroatom functional group, so the only pyrazolidines accessible using this approach are those with alkyl or aryl substituents at the C-3. 


\section{Experimental Section}

General Procedures. Melting points were determined using a Kofler hot-stage apparatus and are uncorrected. Combustion analyses were performed by MEDAC Ltd. (Englefield Green, Surrey). Accurate mass measurements were carried out by the EPSRC National Mass Spectrometry Service Centre (University of Wales Swansea). Infrared spectra were recorded using a PerkinElmer 1710 FT-IR spectrometer. ${ }^{1} \mathrm{H}$ NMR spectra were obtained at $400 \mathrm{MHz}$ on a JEOL JNMEX400 or at $300 \mathrm{MHz}$ on a JEOL JNM-LA300 spectrometer. ${ }^{13} \mathrm{C}$ NMR spectra were recorded at $100 \mathrm{MHz}$ or at $75 \mathrm{MHz}$, respectively, on the same instruments. Low resolution mass spectra were recorded on a VG Micromass VG20-250 spectrometer or by the EPSRC National Mass Spectrometry Service Centre (University of Wales Swansea). Crystallographic measurements were carried out by the EPSRC X-Ray Crystallography Service (University of Southampton). All reagents were purified by distillation or recrystallization where appropriate, or according to standard procedures. ${ }^{19}$ Anhydrous toluene was obtained commercially. Column chromatography was carried out using Fluka Silica Gel 60 (220-440 mesh). Preparative HPLC was performed using a Waters Delta Prep 3000 instrument fitted with a Jones Apex 2 silica column (5 micron, $25 \mathrm{~cm} \times 21 \mathrm{~mm}$ i.d.).

syn- and anti-1-Acetyl-4-methoxycarbonyl-2-methyl-3-phenyltetrahydropyrazoles (10) and (11). Benzaldehyde (1.20 g, $11.31 \mathrm{mmol}), N^{1}$-acetyl- $N^{2}$-methylhydrazine $(\mathbf{9}, 1.05 \mathrm{~g}, 11.92 \mathrm{mmol})$ and methyl propenoate $(1.53 \mathrm{~g}, 17.77 \mathrm{mmol})$ in toluene $(70 \mathrm{~mL})$ were placed in a flask fitted with a Dean-Stark trap. After heating at reflux for $72 \mathrm{~h}$, the solvent was removed using a rotary evaporator and the crude product purified by column chromatography (hexane:ethyl acetate 2:1 $\mathrm{v} / \mathrm{v})$ to give the syn-diastereoisomer $10(0.50 \mathrm{~g}, 17 \%)$ as a white solid, m.p. $47-50^{\circ} \mathrm{C}$. The other diastereoisomer was also isolated $(0.63 \mathrm{~g}, 22 \%)$ containing traces of an impurity (tentatively assigned as the 5-methoxycarbonyl isomer 12). This was removed by further purification using normal-phase preparative HPLC $\left(0.375 \%\right.$ methanol in $\left.\mathrm{CH}_{2} \mathrm{Cl}_{2}, \mathrm{v} / \mathrm{v}\right)$ to give the antidiastereoisomer $11(0.17 \mathrm{~g}, 6 \%)$ as a white solid, m.p. $79-81^{\circ} \mathrm{C}$. Also isolated from the reaction mixture were $N^{1}$-(2-methoxycarbonylethyl)- $N^{1}$-methyl- $N^{2}$-acetylhydrazine 13, as an oil $(0.90 \mathrm{~g}$, $33 \%$ ), and 1,4-diacetyl-2,5-dimethyl-3,6-diphenylhexahydrotetrazine $14(0.53 \mathrm{~g}, 27 \%)$, as a white solid, m.p. $80-82^{\circ} \mathrm{C}$.

syn- Isomer (10): Found: $\mathrm{C}, 63.99 ; \mathrm{H}, 6.96 ; \mathrm{N}, 10.68 \% ; \mathrm{M}^{+}, 262.1318 . \mathrm{C}_{14} \mathrm{H}_{18} \mathrm{~N}_{2} \mathrm{O}_{3}$ requires $\mathrm{C}$, 64.11; H, 6.92; N, 10.67\%; M, 262.1317; $v_{\max }(\mathrm{KBr}) / \mathrm{cm}^{-1} 2955,2380,1728,1646,1437,1382$, 1316, 1213, 1134, 1094, 732 and 697; $\delta_{\mathrm{H}}\left(300 \mathrm{MHz} ; \mathrm{CDCl}_{3}\right) 2.16\left(3 \mathrm{H}, \mathrm{s}, \mathrm{CH}_{3} \mathrm{CO}\right), 2.74(3 \mathrm{H}, \mathrm{s}$, $\left.\mathrm{CH}_{3} \mathrm{~N}\right), 3.35\left(3 \mathrm{H}, \mathrm{s}, \mathrm{CO}_{2} \mathrm{CH}_{3}\right), 3.76(1 \mathrm{H}, \mathrm{dd}, J 9.7,11.6 \mathrm{~Hz}, 5-\mathrm{CHH}), 3.94\left(1 \mathrm{H}, \mathrm{m}, \mathrm{CHCO}_{2}\right), 4.35$ $\left(1 \mathrm{H}, \mathrm{d}, J 7.7 \mathrm{~Hz}, \mathrm{C}_{6} \mathrm{H}_{5} \mathrm{CH}\right), 4.45(1 \mathrm{H}, \mathrm{dd}, J 7.5,11.6 \mathrm{~Hz}, 5-\mathrm{CH} H), 7.05-7.14(2 \mathrm{H}, \mathrm{m}, \mathrm{Ar}-\mathrm{H})$ and 7.23-7.33 (3H, m, Ar-H); $\delta_{\mathrm{C}}\left(75 \mathrm{MHz} ; \mathrm{CDCl}_{3}\right) 21.08\left(\mathrm{CH}_{3} \mathrm{CO}\right), 44.45\left(5-\mathrm{CH}_{2}\right), 46.17\left(\mathrm{CH}_{3} \mathrm{~N}\right)$, $47.84\left(\mathrm{CHCO}_{2}\right), 51.77\left(\mathrm{CO}_{2} \mathrm{CH}_{3}\right), 72.36\left(\mathrm{C}_{6} \mathrm{H}_{5} \mathrm{CH}\right), 126.70,127.95$ and $128.48(\mathrm{Ar}-\mathrm{CH}), 137.90$ (Ar-C), 170.06 and $171.45(\mathrm{C}=\mathrm{O}) ; \mathrm{m} / z$ (EI) $262\left(\mathrm{M}^{+}, 7 \%\right), 219$ (100), 187, 159, 115, 91, 77, 57 and 43. 
anti- Isomer (11): Found: C, 64.12; H, 6.94; N, 10.61\%; $\mathrm{MH}^{+}, 263.1396 . \mathrm{C}_{14} \mathrm{H}_{18} \mathrm{~N}_{2} \mathrm{O}_{3}$ requires $\mathrm{C}, 64.11 ; \mathrm{H}, 6.92 ; \mathrm{N}, 10.67 \%$; MH, 263.1396; $v_{\max }(\mathrm{KBr}) / \mathrm{cm}^{-1} 3456,2956,2889,1738,1656$, 1464, 1412, 1382, 1208, 1053, 726 and 701; $\delta_{\mathrm{H}}\left(300 \mathrm{MHz} ; \mathrm{CDCl}_{3}\right) 2.17\left(3 \mathrm{H}, \mathrm{s}, \mathrm{CH}_{3} \mathrm{CO}\right), 2.63$ $\left(3 \mathrm{H}, \mathrm{s}, \mathrm{CH}_{3} \mathrm{~N}\right), 3.45\left(1 \mathrm{H}, \mathrm{m}, \mathrm{CHCO}_{2}\right), 3.76\left(3 \mathrm{H}, \mathrm{s}, \mathrm{CO}_{2} \mathrm{CH}_{3}\right), 3.86(1 \mathrm{H}, \mathrm{m}, 5-\mathrm{CHH}), 4.43(1 \mathrm{H}, \mathrm{m}$, 5-CHH), $4.47\left(1 \mathrm{H}, \mathrm{d}, J 5.9 \mathrm{~Hz}, \mathrm{C}_{6} \mathrm{H}_{5} \mathrm{CH}\right)$ and 7.27-7.37 (5H, m, Ar-H); $\delta_{\mathrm{C}}\left(75 \mathrm{MHz} ; \mathrm{CDCl}_{3}\right)$ $20.83\left(\mathrm{CH}_{3} \mathrm{CO}\right), 44.51\left(\mathrm{CH}_{3} \mathrm{~N}\right), 45.02\left(5-\mathrm{CH}_{2}\right), 50.87\left(\mathrm{CHCO}_{2}\right), 52.62\left(\mathrm{CO}_{2} \mathrm{CH}_{3}\right), 73.51$ $\left(\mathrm{C}_{6} \mathrm{H}_{5} \mathrm{CH}\right), 126.68,127.90$ and $128.77(\mathrm{Ar}-\mathrm{CH}), 139.86(\mathrm{Ar}-\mathrm{C}), 171.05$ and $172.72(\mathrm{C}=\mathrm{O}) ; \mathrm{m} / z$ (EI) $263\left(\mathrm{MH}^{+}, 10 \%\right), 219,187,159,131,117,115,91,77,57$ and 43 (100).

Hydrazine (13): major rotational isomer: $\delta_{\mathrm{H}}\left(300 \mathrm{MHz} ; \mathrm{CDCl}_{3}\right) 2.06\left(3 \mathrm{H}, \mathrm{s}, \mathrm{CH}_{3} \mathrm{CO}\right), 2.48-2.61$ $\left(2 \mathrm{H}, \mathrm{m}, \mathrm{CH}_{2}\right), 2.59\left(3 \mathrm{H}, \mathrm{s}, \mathrm{CH}_{3} \mathrm{~N}\right), 2.86-3.07\left(2 \mathrm{H}, \mathrm{m}, \mathrm{CH}_{2}\right), 3.69\left(3 \mathrm{H}, \mathrm{s}, \mathrm{CO}_{2} \mathrm{CH}_{3}\right)$ and $6.50(1 \mathrm{H}$, br. s, NH); $\delta_{\mathrm{C}}\left(75 \mathrm{MHz} ; \mathrm{CDCl}_{3}\right) 19.63\left(\mathrm{CH}_{3} \mathrm{CO}\right), 32.34\left(\mathrm{CH}_{2}\right), 47.37\left(\mathrm{CH}_{3} \mathrm{~N}\right), 51.84\left(\mathrm{CH}_{2}\right)$, $55.53\left(\mathrm{CO}_{2} \mathrm{CH}_{3}\right), 172.36$ and $174.84(\mathrm{C}=\mathrm{O})$; minor rotational isomer: $\delta_{\mathrm{H}}\left(300 \mathrm{MHz} ; \mathrm{CDCl}_{3}\right) 1.90$ $\left(3 \mathrm{H}, \mathrm{s}, \mathrm{CH}_{3} \mathrm{CO}\right), 2.48-2.61\left(2 \mathrm{H}, \mathrm{m}, \mathrm{CH}_{2}\right), 2.66\left(3 \mathrm{H}, \mathrm{s}, \mathrm{CH}_{3} \mathrm{~N}\right), 2.86-3.07\left(2 \mathrm{H}, \mathrm{m}, \mathrm{CH}_{2}\right), 3.69(3 \mathrm{H}$, s, $\left.\mathrm{CO}_{2} \mathrm{CH}_{3}\right)$ and $6.54(1 \mathrm{H}$, br. s, $\mathrm{NH}) ; \delta_{\mathrm{C}}\left(75 \mathrm{MHz} ; \mathrm{CDCl}_{3}\right) 21.48\left(\mathrm{CH}_{3} \mathrm{CO}\right), 32.40\left(\mathrm{CH}_{2}\right), 46.17$ $\left(\mathrm{CH}_{3} \mathrm{~N}\right), 51.81\left(\mathrm{CH}_{2}\right), 54.01\left(\mathrm{CO}_{2} \mathrm{CH}_{3}\right), 168.62$ and $173.20(\mathrm{C}=\mathrm{O}) ; m / z(\mathrm{EI}) 174\left(\mathrm{M}^{+}, 1 \%\right), 143$, 131, 116, 101, 59 (100) and 43.

Tetrazine (14): Found: C, 68.18; H, 6.87; N, 15.86\%; $\mathrm{MH}^{+}, 353.1986 . \mathrm{C}_{20} \mathrm{H}_{24} \mathrm{~N}_{4} \mathrm{O}_{2}$ requires C, 68.16; H, 6.86; N, 15.89; MH, 353.1977; $v_{\max }(\mathrm{KBr}) / \mathrm{cm}^{-1} 3020,2975,1682,1602,1573,1462$, 1411, 1398, 1142, 995, 762 and 698; $\delta_{\mathrm{H}}\left(300 \mathrm{MHz} ; \mathrm{CDCl}_{3}\right) 2.46\left(6 \mathrm{H}, \mathrm{s}, \mathrm{CH}_{3} \mathrm{CO}\right), 3.34(6 \mathrm{H}, \mathrm{s}$, $\left.\mathrm{CH}_{3} \mathrm{~N}\right), 7.34-7.44(6 \mathrm{H}, \mathrm{m}, \mathrm{Ar}-\mathrm{H})$ and 7.59-7.72 (6H, m, Ar-H and $\left.\mathrm{NCHN}\right) ; \delta_{\mathrm{C}}\left(75 \mathrm{MHz} ; \mathrm{CDCl}_{3}\right)$ $21.59\left(\mathrm{CH}_{3} \mathrm{CO}\right), 27.47\left(\mathrm{CH}_{3} \mathrm{~N}\right), 126.95,128.74$ and $129.62(\mathrm{Ar}-\mathrm{CH}), 134.81$ (Ar-C), 138.72 $(\mathrm{NCHN})$ and $172.88(\mathrm{C}=\mathrm{O}) ; \mathrm{m} / z(\mathrm{EI}) 176\left(\mathrm{M} / 2^{+}, 5 \%\right), 133,90,89,77,63,51$ and $43(100)$.

Crystallographic data for (11): $\mathrm{C}_{14} \mathrm{H}_{18} \mathrm{~N}_{2} \mathrm{O}_{3}, M=262.30$, triclinic, $a=7.5758(3), b=$ 9.5502(4), $c=9.8576(3) \AA, a=84.009(2), b=86.769(2), g=74377(2)^{\circ}, U=682.80(4) \AA^{3}, T=$ $150(2) \mathrm{K}$, space group $P_{-1}$, monochromatic Mo-Ka radiation, $l=0.71073 \AA, Z=2, D_{c}=1.276$ $\mathrm{Mg} \mathrm{m}^{-3}, F(000)=280$, colorless block, dimensions 0.4 x $0.3 \times 0.3 \mathrm{~mm}, m(\mathrm{Mo}-\mathrm{Ka})=0.090$ $\mathrm{mm}^{-1}, 3.07<2 q<25.02^{\circ}, 6798$ reflections measured, 2386 unique reflections. The structure was solved by direct methods and refined by full-matrix least-squares on $F^{2}$. The final cycle (for 245 parameters) converged with $w R 2=0.0912$ (for all data) and $R 1=0.0363\left[F^{2}>2 s\left(F^{2}\right)\right]$.

syn- and anti-1-Acetyl-4-cyano-2-methyl-3-phenyltetrahydropyrazoles (15) and (16). The procedure described for the synthesis of 1-acetyl-4-methoxycarbonyl-2-methyl-3phenyltetrahydropyrazoles 10 and 11 was followed, but using benzaldehyde (0.94 g, $8.85 \mathrm{mmol})$, $N^{1}$-acetyl- $N^{2}$-methylhydrazine $9(0.76 \mathrm{~g}, 8.63 \mathrm{mmol})$, propenonitrile $(0.93 \mathrm{~g}, 17.47 \mathrm{mmol})$ and toluene $(40 \mathrm{~mL})$. Column chromatography afforded the pure syn- diastereoisomer 15 (0.29 g, $15 \%$ ), as a pale yellow solid, m.p. $150-153^{\circ} \mathrm{C}$. The anti-diastereoisomer 16 was also isolated as a yellow solid $(0.20 \mathrm{~g}, 10 \%)$ containing traces of an impurity (tentatively assigned as the 5-cyano isomer).

syn-Isomer (15): Found: $\mathrm{C}, 68.09 ; \mathrm{H}, 6.64 ; \mathrm{N}, 18.06 \% ; \mathrm{M}^{+}, 229.1216 . \mathrm{C}_{13} \mathrm{H}_{15} \mathrm{~N}_{3} \mathrm{O}$ requires $\mathrm{C}$, 68.10; H, 6.59; N, 18.32\%; M, 229.1215; $v_{\max }(\mathrm{KBr}) / \mathrm{cm}^{-1} 2998,2964,2895,2246,1656,1470$, 1453, 1444, 1414, 1373, 758 and 712; $\delta_{\mathrm{H}}\left(300 \mathrm{MHz} ; \mathrm{CDCl}_{3}\right) 2.21\left(3 \mathrm{H}, \mathrm{s}, \mathrm{CH}_{3} \mathrm{CO}\right), 2.72(3 \mathrm{H}, \mathrm{s}$, 
$\left.\mathrm{CH}_{3} \mathrm{~N}\right), 3.78(1 \mathrm{H}$, ddd, $J$ 5.6, 6.6, 8.2 Hz, CHCN), $3.87(1 \mathrm{H}, \mathrm{dd}, J$ 8.2, $11.4 \mathrm{~Hz}, 5-\mathrm{CHH}), 4.32$ $\left(1 \mathrm{H}, \mathrm{d}, J 6.6 \mathrm{~Hz}, \mathrm{C}_{6} \mathrm{H}_{5} \mathrm{CH}\right), 4.41(1 \mathrm{H}, \mathrm{dd}, J 5.6,11.4 \mathrm{~Hz}, 5-\mathrm{CH} H), 7.25-7.27(2 \mathrm{H}, \mathrm{m}, \mathrm{Ar}-\mathrm{H})$ and 7.31-7.43 (3H, m, Ar-H); $\delta_{\mathrm{C}}\left(75 \mathrm{MHz} ; \mathrm{CDCl}_{3}\right) 20.90\left(\mathrm{CH}_{3} \mathrm{CO}\right), 34.90(\mathrm{CHCN}), 45.83\left(\mathrm{CH}_{3} \mathrm{~N}\right)$, $46.50\left(5-\mathrm{CH}_{2}\right), 72.29\left(\mathrm{C}_{6} \mathrm{H}_{5} \mathrm{CH}\right), 117.07(\mathrm{CN}), 127.00,128.88$ and $128.94(\mathrm{Ar}-\mathrm{CH}), 135.85(\mathrm{Ar}-$ C) and $172.09(\mathrm{C}=\mathrm{O}) ; \mathrm{m} / z(\mathrm{EI}) 229\left(\mathrm{M}^{+}, 5 \%\right), 186(100), 159,142,133,115,91,77,57$ and 43.

anti-Isomer (16): Found: C, 67.90; H, 6.57; N, 18.26\%; $\mathrm{M}^{+}, 229.1226 . \mathrm{C}_{13} \mathrm{H}_{15} \mathrm{~N}_{3} \mathrm{O}$ requires $\mathrm{C}$, 68.10; H, 6.59; N, 18.32\%; M, 229.1215; $v_{\max }(\mathrm{KBr}) / \mathrm{cm}^{-1} 3035,2994,2963,2922,2883,2802$, 2247, 1662, 1496, 1398, 1344, 1319, 1216, 1110, 768, 738 and 702; $\delta_{\mathrm{H}}\left(300 \mathrm{MHz} ; \mathrm{CDCl}_{3}\right) 2.14$ $\left(3 \mathrm{H}, \mathrm{s}, \mathrm{CH}_{3} \mathrm{CO}\right), 2.75\left(3 \mathrm{H}, \mathrm{s}, \mathrm{CH}_{3} \mathrm{~N}\right), 3.44(1 \mathrm{H}, \mathrm{m}, \mathrm{CHCN}), 3.84(1 \mathrm{H}, \mathrm{dd}, J$ 7.1, $11.9 \mathrm{~Hz}, 5-$ $\mathrm{CHH}), 4.46\left(1 \mathrm{H}, \mathrm{d}, J 6.0 \mathrm{~Hz}, \mathrm{C}_{6} \mathrm{H}_{5} \mathrm{CH}\right), 4.49(1 \mathrm{H}, \mathrm{m}, 5-\mathrm{CH} H), 7.26-7.32(2 \mathrm{H}, \mathrm{m}, \mathrm{Ar}-\mathrm{H})$ and 7.34-7.42 $(3 \mathrm{H}, \mathrm{m}, \mathrm{Ar}-\mathrm{H}) ; \delta_{\mathrm{C}}\left(75 \mathrm{MHz} ; \mathrm{CDCl}_{3}\right) 20.82\left(\mathrm{CH}_{3} \mathrm{CO}\right), 34.96(\mathrm{CHCN}), 44.48\left(\mathrm{CH}_{3} \mathrm{~N}\right)$, $46.04\left(5-\mathrm{CH}_{2}\right), 74.21\left(\mathrm{C}_{6} \mathrm{H}_{5} \mathrm{CH}\right), 119.38(\mathrm{CN}), 126.52,128.74$ and $129.11(\mathrm{Ar}-\mathrm{CH}), 137.26$ (ArC) and $171.55(\mathrm{C}=\mathrm{O}) ; \mathrm{m} / \mathrm{z}(\mathrm{EI}) 229\left(\mathrm{M}^{+}, 1 \%\right), 186,143,133,115,91,77,57$ and $43(100)$.

anti, anti-1-Acetyl-4,5-bis(methoxycarbonyl)-2-methyl-3-phenyltetrahydropyrazole (17). The procedure described for the synthesis of 1-acetyl-4-methoxycarbonyl-2-methyl-3phenyltetrahydropyrazoles 10 and 11 was followed, but using benzaldehyde $(0.61 \mathrm{~g}, 5.71 \mathrm{mmol})$, $N^{1}$-acetyl- $N^{2}$-methylhydrazine $9(0.51 \mathrm{~g}, 5.79 \mathrm{mmol})$, dimethyl fumarate $(1.68 \mathrm{~g}, 11.66 \mathrm{mmol})$ and toluene $(40 \mathrm{~mL})$. Column chromatography afforded the title compound $\mathbf{1 7}$ as a white solid $(0.69 \mathrm{~g}, 38 \%)$, m.p. $152-154^{\circ} \mathrm{C}$, along with a mixture $(0.49 \mathrm{~g}, 27 \%)$ of the title compound $(21 \%)$ and an isomer tentatively assigned as the syn, anti-diastereoisomer $18(6 \%)$.

anti, anti-Diastereoisomer (17): Found: $\mathrm{C}, 59.89 ; \mathrm{H}, 6.27 ; \mathrm{N}, 8.71 \% ; \mathrm{M}^{+}, 320.1368$. $\mathrm{C}_{16} \mathrm{H}_{20} \mathrm{~N}_{2} \mathrm{O}_{5}$ requires $\mathrm{C}, 59.99 ; \mathrm{H}, 6.29 ; \mathrm{N}, 8.74 \% ; \mathrm{M}, 320.1372 ; v_{\max }(\mathrm{KBr}) / \mathrm{cm}^{-1} 2991,2963$, 2361, 1765, 1732, 1654, 1435, 1409, 1215, 764 and 708; $\delta_{\mathrm{H}}\left(300 \mathrm{MHz} ; \mathrm{CDCl}_{3}\right) 2.27(6 \mathrm{H}, \mathrm{s}$, $\mathrm{CH}_{3} \mathrm{CO}$ and $\mathrm{CH}_{3} \mathrm{~N}$ ), 3.71 and 3.81 (each $\left.3 \mathrm{H}, \mathrm{s}, \mathrm{CO}_{2} \mathrm{CH}_{3}\right), 4.03(1 \mathrm{H}$, dd, $J 8.3,10.8 \mathrm{~Hz}$, 4$\left.\mathrm{CHCO}_{2}\right), 4.58\left(1 \mathrm{H}, \mathrm{d}, J 10.8 \mathrm{~Hz}, \mathrm{C}_{6} \mathrm{H}_{5} \mathrm{CH}\right), 5.16\left(1 \mathrm{H}, \mathrm{d}, J 8.3 \mathrm{~Hz}, 5-\mathrm{CHCO}_{2}\right)$ and 7.33-7.43 $(5 \mathrm{H}$, m, Ar-H); $\delta_{\mathrm{C}}\left(75 \mathrm{MHz} ; \mathrm{CDCl}_{3}\right) 20.95\left(\mathrm{CH}_{3} \mathrm{CO}\right), 39.75\left(\mathrm{CH}_{3} \mathrm{~N}\right), 49.79\left(4-\mathrm{CHCO}_{2}\right), 52.84$ and $52.97\left(\mathrm{CO}_{2} \mathrm{CH}_{3}\right), 62.33\left(5-\mathrm{CHCO}_{2}\right), 71.90\left(\mathrm{C}_{6} \mathrm{H}_{5} \mathrm{CH}\right), 128.82,128.83$ and $128.85(\mathrm{Ar}-\mathrm{CH})$, 132.85 (Ar-C), 170.93, 171.00 and $171.18(\mathrm{C}=\mathrm{O}) ; \mathrm{m} / \mathrm{z}(\mathrm{EI}) 320\left(\mathrm{M}^{+}, 12 \%\right), 278,245,217,185$, $159,115,91,77,59$ and 43 (100).

syn, syn-1-Acetyl-4,5-bis-(methoxycarbonyl)-2-methyl-3-phenyltetrahydropyrazole (19). The procedure described for the synthesis of 1-acetyl-4-methoxycarbonyl-2-methyl-3phenyltetrahydropyrazoles 10 and 11 was followed, but using benzaldehyde (0.56 g, $5.31 \mathrm{mmol})$, $N^{1}$-acetyl- $N^{2}$-methylhydrazine $9(0.47 \mathrm{~g}, 5.33 \mathrm{mmol})$, dimethyl maleate $(1.66 \mathrm{~g}, 11.52 \mathrm{mmol})$ and toluene $(40 \mathrm{~mL})$. Column chromatography afforded an inseparable mixture of stereoisomeric tetrahydropyrazoles $(0.43 \mathrm{~g}, 25 \%)$, and the title compound $19(0.18 \mathrm{~g}, 11 \%)$ as a white solid, m.p. 155-158 ${ }^{\circ}$; Found: C, 59.78; H, 6.32; N, 8.58\%; $\mathrm{M}^{+}, 320.1372 . \mathrm{C}_{16} \mathrm{H}_{20} \mathrm{~N}_{2} \mathrm{O}_{5}$ requires $\mathrm{C}$, 59.99; H, 6.29; N, 8.74\%; M, 320.1372; $v_{\max }(\mathrm{KBr}) / \mathrm{cm}^{-1} 3007,2953,1765,1747,1641,1494$, $1455,1433,1377,1356,1342,1198,1168,1110,741$ and $700 ; \delta_{\mathrm{H}}\left(300 \mathrm{MHz} ; \mathrm{CDCl}_{3}\right) 2.33(3 \mathrm{H}$, s, $\left.\mathrm{CH}_{3} \mathrm{CO}\right), 2.78\left(3 \mathrm{H}, \mathrm{s}, \mathrm{CH}_{3} \mathrm{~N}\right), 3.53$ and 3.66 (each $\left.3 \mathrm{H}, \mathrm{s}, \mathrm{CO}_{2} \mathrm{CH}_{3}\right), 4.23(1 \mathrm{H}, \mathrm{dd}, J 7.9,10.1$ $\left.\mathrm{Hz}, 4-\mathrm{CHCO}_{2}\right), 4.50\left(1 \mathrm{H}, \mathrm{d}, J 7.9 \mathrm{~Hz}, \mathrm{C}_{6} \mathrm{H}_{5} \mathrm{CH}\right), 4.85\left(1 \mathrm{H}, \mathrm{d}, J 10.1 \mathrm{~Hz}, 5-\mathrm{CHCO}_{2}\right)$ and 7.24- 
$7.38(5 \mathrm{H}, \mathrm{m}, \mathrm{Ar}-\mathrm{H}) ; \delta_{\mathrm{C}}\left(75 \mathrm{MHz} ; \mathrm{CDCl}_{3}\right) 21.18\left(\mathrm{CH}_{3} \mathrm{CO}\right), 45.23\left(\mathrm{CH}_{3} \mathrm{~N}\right), 51.18\left(4-\mathrm{CHCO}_{2}\right)$, 51.98 and $52.24\left(\mathrm{CO}_{2} \mathrm{CH}_{3}\right), 59.00\left(5-\mathrm{CHCO}_{2}\right), 71.65\left(\mathrm{C}_{6} \mathrm{H}_{5} \mathrm{CH}\right), 127.97,128.01$ and 128.09 (Ar$\mathrm{CH}), 136.08$ (Ar-C), 167.93, 168.74 and $169.57(\mathrm{C}=\mathrm{O}) ; m / z(\mathrm{EI}) 321\left(\mathrm{MH}^{+}, 2 \%\right), 278,219,159$, 133, 115, 91, 77, 59 and 43 (100).

anti, syn-1-Acetyl-2-methyl-3,5-diphenyloctahydropyrrolo[3,4-c]pyrazole-4,6-dione (20). The procedure described for the synthesis of 1-acetyl-4-methoxycarbonyl-2-methyl-3phenyltetrahydropyrazoles 10 and 11 was followed, but using benzaldehyde ( $0.63 \mathrm{~g}, 5.90 \mathrm{mmol})$, $N^{1}$-acetyl- $N^{2}$-methylhydrazine $9(0.55 \mathrm{~g}, 6.24 \mathrm{mmol}), N$-phenylmaleimide $(2.05 \mathrm{~g}, 11.84 \mathrm{mmol})$ and toluene $(40 \mathrm{~mL})$. Column chromatography (hexane: ethyl acetate $3: 2 \mathrm{v} / \mathrm{v}$ ) afforded the title compound 20 (0.08 g, 4\%) as a yellow solid, m.p. 239-241 ${ }^{\circ} \mathrm{C}$; Found: C, 68.43; H, 5.44; N, $11.58 \% ; \mathrm{M}^{+}, 349.1422 . \mathrm{C}_{20} \mathrm{H}_{19} \mathrm{~N}_{3} \mathrm{O}_{3}$ requires $\mathrm{C}, 68.75 ; \mathrm{H}, 5.44 ; \mathrm{N}, 12.02 \% ; \mathrm{M}, 349.1426 ; v_{\max }$ $(\mathrm{KBr}) / \mathrm{cm}^{-1} 1719,1653,1499,1386,1199,1158,746$ and 696; $\delta_{\mathrm{H}}\left(300 \mathrm{MHz} ; \mathrm{CDCl}_{3}\right) 2.03(3 \mathrm{H}$, $\left.\mathrm{s}, \mathrm{CH}_{3} \mathrm{CO}\right), 2.87\left(3 \mathrm{H}, \mathrm{s}, \mathrm{CH}_{3} \mathrm{~N}\right), 4.02\left(1 \mathrm{H}, \mathrm{d}, J 8.6 \mathrm{~Hz}, \mathrm{C}_{6} \mathrm{H}_{5} \mathrm{CHCHCO}\right), 4.73\left(1 \mathrm{H}, \mathrm{s}, \mathrm{C}_{6} \mathrm{H}_{5} \mathrm{CH}\right)$, $5.80(1 \mathrm{H}, \mathrm{d}, J 8.6 \mathrm{~Hz}, \mathrm{NCHCO})$ and 7.18-7.53 $(10 \mathrm{H}, \mathrm{m}, \mathrm{Ar}-\mathrm{H}) ; \delta_{\mathrm{C}}\left(75 \mathrm{MHz} ; \mathrm{CDCl}_{3}\right) 20.88$ $\left(\mathrm{CH}_{3} \mathrm{CO}\right), 47.55\left(\mathrm{CH}_{3} \mathrm{~N}\right), 53.11\left(\mathrm{C}_{6} \mathrm{H}_{5} \mathrm{CHCHCO}\right) 58.42(\mathrm{NCHCO}), 73.07\left(\mathrm{C}_{6} \mathrm{H}_{5} \mathrm{CH}\right), 125.82$, 125.99 128.28, 129.07, 129.12 and $129.39(\mathrm{Ar}-\mathrm{CH}), 131.51$ and 139.67 (Ar-C), $172.99(2 \mathrm{x}$ $\mathrm{C}=\mathrm{O})$ and $175.80(\mathrm{C}=\mathrm{O}) ; \mathrm{m} / z(\mathrm{EI}) 350\left(\mathrm{MH}^{+}, 82 \%\right), 349(100), 307,159,133,115,91,77$ and 43.

syn- and anti-1-Acetyl-4-methoxycarbonyl-2-methyl-3-(2-propyl)tetrahydropyrazoles (21) and (22). The procedure described for the synthesis of 1-acetyl-4-methoxycarbonyl-2-methyl-3phenyltetrahydropyrazoles $\mathbf{1 0}$ and 11 was followed, but using 2-methylpropanal $(0.24 \mathrm{~g}, 3.30$ mmol), $N^{1}$-acetyl- $N^{2}$-methylhydrazine $9(0.50 \mathrm{~g}, 5.67 \mathrm{mmol})$, methyl propenoate $(1.00 \mathrm{~g}, 11.66$ $\mathrm{mmol})$ and toluene $(50 \mathrm{~mL})$, and heating at reflux for $16 \mathrm{~h}$. Column chromatography (hexane:ethyl acetate 1:1 v/v) yielded syn-diastereoisomer 21 as a yellow oil (0.19 g, 25\%), and a fraction that was purified by normal-phase preparative $\mathrm{HPLC}\left(\mathrm{CH}_{3} \mathrm{CN}\right.$ : water $\left.30 \% \mathrm{v} / \mathrm{v}\right)$ to afford the anti-diastereoisomer $22(0.09 \mathrm{~g}, 11 \%)$ and the 5-methoxycarbonyl isomer $23(0.03 \mathrm{~g}$, $1 \%$ ), both as yellow oils. $N^{1}$-(2-Methoxycarbonylethyl)- $N^{1}$-methyl- $N^{2}$-acetylhydrazine 13 was also isolated $(0.45 \mathrm{~g}, 46 \%)$.

syn-Diastereoisomer (21). Found: $\mathrm{M}^{+}$, 228.1474. $\mathrm{C}_{11} \mathrm{H}_{20} \mathrm{~N}_{2} \mathrm{O}_{3}$ requires $\mathrm{M}, 228.1474$; $v_{\max }$ $\left(\mathrm{CHCl}_{3}\right) / \mathrm{cm}^{-1} 3670,3441,3013,2925,1740,1647,1439,1373,1245,1185$ and 1124; $\delta_{\mathrm{H}}(300$ $\left.\mathrm{MHz} \mathrm{CDCl}_{3}\right) 0.83$ and $0.94\left(\right.$ each $\left.3 \mathrm{H}, \mathrm{d}, J 6.6 \mathrm{~Hz}, \mathrm{CH}_{3} \mathrm{CH}\right), 1.70(1 \mathrm{H}, \mathrm{m}, \mathrm{CHMe}), 2.20(3 \mathrm{H}, \mathrm{s}$, $\left.\mathrm{CH}_{3} \mathrm{CON}\right), 2.59\left(3 \mathrm{H}, \mathrm{s}, \mathrm{CH}_{3} \mathrm{~N}\right), 3.02\left(1 \mathrm{H}, \mathrm{m}, \mathrm{CHCHMe}_{2}\right), 3.67(1 \mathrm{H}, \mathrm{m}, 5-\mathrm{CHH}), 3.72(3 \mathrm{H}, \mathrm{s}$, $\left.\mathrm{CO}_{2} \mathrm{CH}_{3}\right), 3.76\left(1 \mathrm{H}, \mathrm{m}, \mathrm{CHCO}_{2}\right)$ and $4.20(1 \mathrm{H}, \mathrm{m}, 5-\mathrm{CH}) ; \delta_{\mathrm{C}}\left(100 \mathrm{MHz} ; \mathrm{CDCl}_{3}\right) 18.02$ $\left.\left(\mathrm{CH}_{3} \mathrm{CH}\right), 20.75\left(\mathrm{CH}_{3} \mathrm{CON}\right), 21.09\left(\mathrm{CH}_{3} \mathrm{CH}\right), 29.23 \mathrm{CHMe}_{2}\right), 44.80\left(5-\mathrm{CH}_{2}\right), 44.98\left(\mathrm{CHCO}_{2}\right)$, $46.57\left(\mathrm{CH}_{3} \mathrm{~N}\right), 52.00\left(\mathrm{CO}_{2} \mathrm{CH}_{3}\right), 73.69\left(\mathrm{CHCHMe}_{2}, 170.51\right.$ and $171.06(\mathrm{C}=\mathrm{O}) ; \mathrm{m} / z(\mathrm{EI}) 228\left(\mathrm{M}^{+}\right.$, $13 \%), 197,185$ (100), 143, 125, 83 and 43.

anti-Diastereoisomer (22): Found: $\mathrm{M}^{+}$, 228.1474. $\mathrm{C}_{11} \mathrm{H}_{20} \mathrm{~N}_{2} \mathrm{O}_{3}$ requires $\mathrm{M}, 228.1474$; $v_{\max }$ $\left(\mathrm{CHCl}_{3}\right) / \mathrm{cm}^{-1} 2963,1734,1637,1498,1369,1257$ and $1185 ; \delta_{\mathrm{H}}\left(400 \mathrm{MHz} ; \mathrm{CDCl}_{3}\right) 0.93(3 \mathrm{H}, \mathrm{d}$, $\left.J 6.4 \mathrm{~Hz}, \mathrm{CH}_{3} \mathrm{CH}\right), 0.99\left(3 \mathrm{H}, \mathrm{d}, J 6.8 \mathrm{~Hz}, \mathrm{CH}_{3} \mathrm{CH}\right), 1.50(1 \mathrm{H}, \mathrm{m}, \mathrm{CHMe}), 2.16(3 \mathrm{H}, \mathrm{s}$, $\left.\mathrm{CH}_{3} \mathrm{CON}\right), 2.60\left(3 \mathrm{H}, \mathrm{s}, \mathrm{CH}_{3} \mathrm{~N}\right), 2.94\left(1 \mathrm{H}, \mathrm{dd}, J 3.9,8.8 \mathrm{~Hz}, \mathrm{CHCHMe}_{2}, 3.05\left(1 \mathrm{H}, \mathrm{m}, \mathrm{CHCO}_{2}\right)\right.$, 
$3.68(1 \mathrm{H}, \mathrm{m}, 5-\mathrm{CHH}), 3.75\left(3 \mathrm{H}, \mathrm{s}, \mathrm{CO}_{2} \mathrm{CH}_{3}\right)$ and $4.37(1 \mathrm{H}, \mathrm{m}, 5-\mathrm{CH} H) ; \delta_{\mathrm{C}}\left(100 \mathrm{MHz} ; \mathrm{CDCl}_{3}\right)$ 19.11 and $19.81\left(\mathrm{CH}_{3} \mathrm{CH}\right), 20.57\left(\mathrm{CH}_{3} \mathrm{CON}\right), 32.61\left(\mathrm{CHMe}_{2}\right), 44.22\left(5-\mathrm{CH}_{2}\right), 45.79\left(\mathrm{CH}_{3} \mathrm{~N}\right)$, $47.46\left(\mathrm{CHCO}_{2}\right), 52.49\left(\mathrm{CO}_{2} \mathrm{CH}_{3}\right), 77.07\left(\mathrm{CHCHMe}_{2}\right), 170.88$ and $173.99(\mathrm{C}=\mathrm{O}) ; \mathrm{m} / z(\mathrm{EI}) 228$ $\left(\mathrm{M}^{+}, 24 \%\right), 197,185$ (100), 169, 143, 125, 83 and 43.

5-Methoxycarbonyl isomer (23): Found: $\mathrm{C}, 56.73 ; \mathrm{H}, 8.74 ; \mathrm{N}, 12.00 \% ; \mathrm{M}^{+}, 228.1474$. $\mathrm{C}_{11} \mathrm{H}_{20} \mathrm{~N}_{2} \mathrm{O}_{3} \cdot 0.25 \mathrm{H}_{2} \mathrm{O}$ requires $\mathrm{C}, 56.75 ; \mathrm{H}, 8.87 ; \mathrm{N}, 12.03 \% ; \mathrm{C}_{11} \mathrm{H}_{20} \mathrm{~N}_{2} \mathrm{O}_{3}$ requires $\mathrm{M}, 228.1474$; $v_{\max }\left(\mathrm{CHCl}_{3}\right) / \mathrm{cm}^{-1} 2960,1749,1646,1438,1420$ and 1370; major rotational isomer: $\delta_{\mathrm{H}}(400$ $\left.\mathrm{MHz} ; \mathrm{CDCl}_{3}\right) 0.92\left(3 \mathrm{H}, \mathrm{d}, J 6.4 \mathrm{~Hz}, \mathrm{CH}_{3} \mathrm{CH}\right), 1.05\left(3 \mathrm{H}, \mathrm{d}, J 6.3 \mathrm{~Hz}, \mathrm{CH}_{3} \mathrm{CH}\right), 1.73(1 \mathrm{H}, \mathrm{m}$, $\left.\mathrm{CHMe}_{2}\right), 2.06(1 \mathrm{H}, \mathrm{m}, 4-\mathrm{CHH}), 2.18\left(3 \mathrm{H}, \mathrm{s}, \mathrm{CH}_{3} \mathrm{CON}\right), 2.50\left(3 \mathrm{H}, \mathrm{s}, \mathrm{CH}_{3} \mathrm{~N}\right), 2.53(1 \mathrm{H}, \mathrm{m}, 4-$ $\mathrm{CH} H), 2.66(1 \mathrm{H}, \mathrm{m}, \mathrm{CHCHMe}), 3.74\left(3 \mathrm{H}, \mathrm{s}, \mathrm{CO}_{2} \mathrm{CH}_{3}\right)$ and $4.61\left(1 \mathrm{H}, \mathrm{m}, \mathrm{CHCO}_{2}\right) ; \delta_{\mathrm{C}}(100$ $\left.\mathrm{MHz} ; \mathrm{CDCl}_{3}\right) 20.28\left(\mathrm{CH}_{3} \mathrm{CH}\right), 20.87\left(\mathrm{CH}_{3} \mathrm{CON}\right), 20.96\left(\mathrm{CH}_{3} \mathrm{CH}\right), 28.73\left(\mathrm{CHMe}_{2}\right), 32.61$ (4$\left.\mathrm{CH}_{2}\right), 46.25\left(\mathrm{CH}_{3} \mathrm{~N}\right), 52.40\left(\mathrm{CO}_{2} \mathrm{CH}_{3}\right), 57.63\left(\mathrm{CHCO}_{2}\right), 72.33$ or $73.52\left(\mathrm{CHCHMe}_{2}\right), 170.55$ and $172.36(\mathrm{C}=\mathrm{O})$; minor rotational isomer: $\delta_{\mathrm{H}}\left(400 \mathrm{MHz} ; \mathrm{CDCl}_{3}\right) 0.88$ and $0.96($ each $3 \mathrm{H}, \mathrm{d}, J 6.8$ $\left.\mathrm{Hz}, \mathrm{CH}_{3} \mathrm{CH}\right), 1.33(1 \mathrm{H}, \mathrm{m}, \mathrm{CHMe}), 2.06(1 \mathrm{H}, \mathrm{m}, 4-\mathrm{CHH}), 2.20\left(3 \mathrm{H}, \mathrm{s}, \mathrm{CH}_{3} \mathrm{CON}\right), 2.50(3 \mathrm{H}, \mathrm{s}$, $\left.\mathrm{CH}_{3} \mathrm{~N}\right), 2.53(1 \mathrm{H}, \mathrm{m}, 4-\mathrm{CH} H), 2.66(1 \mathrm{H}, \mathrm{m}, \mathrm{CHCHMe}), 3.76\left(3 \mathrm{H}, \mathrm{s}, \mathrm{CO}_{2} \mathrm{CH}_{3}\right)$ and $4.61(1 \mathrm{H}, \mathrm{m}$, $\left.\mathrm{CHCO}_{2}\right) ; \delta_{\mathrm{C}}\left(100 \mathrm{MHz} ; \mathrm{CDCl}_{3}\right) 19.44\left(\mathrm{CH}_{3} \mathrm{CH}\right), 20.87\left(\mathrm{CH}_{3} \mathrm{CON}\right), 20.96\left(\mathrm{CH}_{3} \mathrm{CH}\right), 30.36$ $\left(\mathrm{CHMe}_{2}\right), 31.27\left(4-\mathrm{CH}_{2}\right), 46.25\left(\mathrm{CH}_{3} \mathrm{~N}\right), 52.40\left(\mathrm{CO}_{2} \mathrm{CH}_{3}\right), 58.16\left(\mathrm{CHCO}_{2}\right), 72.33$ or 73.52 $\left(\mathrm{CHCHMe}_{2}\right), 171.18$ and $172.86(\mathrm{C}=\mathrm{O}) ; \mathrm{m} / z(\mathrm{EI}) 228\left(\mathrm{M}^{+}, 24 \%\right), 197,185(100), 169,143,125$, 83 and $43(100)$.

2-tert-Butoxy-5-(1-methyl-2-acetyl)hydrazino-4-methyl-4,5-dihydrooxazole $\quad$ (25). The procedure described for the synthesis of 1-acetyl-4-methoxycarbonyl-2-methyl-3phenyltetrahydropyrazoles 10 and 11 was followed, but using $(S)$ - $N$-tert-butoxycarbonylalaninal 24 (0.50 g, $2.89 \mathrm{mmol}), N^{1}$-acetyl- $N^{2}$-methylhydrazine 9 (0.38 g, $\left.4.31 \mathrm{mmol}\right)$, methyl propenoate $(0.75 \mathrm{~g}, 8.66 \mathrm{mmol})$ and toluene $(50 \mathrm{~mL})$, and heating at reflux for $16 \mathrm{~h}$. Column chromatography (hexane:ethyl acetate $1: 1 \mathrm{v} / \mathrm{v}$ ) followed by further purification by normal phase HPLC (30\% $\mathrm{CH}_{3} \mathrm{CN}$ in water v/v) afforded only the title compound 25 (0.19 g, 27\%), which was incompletely characterized; $\delta_{\mathrm{H}}\left(400 \mathrm{MHz}, \mathrm{CDCl}_{3}\right) 1.36\left(3 \mathrm{H}, \mathrm{d}, J 7.3 \mathrm{~Hz}, \mathrm{CH}_{3} \mathrm{CH}\right), 1.46(9 \mathrm{H}, \mathrm{s}$, $\left.\mathrm{Me}_{3} \mathrm{C}\right), 2.32\left(3 \mathrm{H}, \mathrm{s}, \mathrm{CH}_{3} \mathrm{CO}\right), 3.19\left(3 \mathrm{H}, \mathrm{s}, \mathrm{CH}_{3} \mathrm{~N}\right), 4.45(1 \mathrm{H}$, br., OCHN$), 5.39(1 \mathrm{H}$, br., $\left.\mathrm{CHNCH}_{3}\right)$ and $7.03\left(1 \mathrm{H}\right.$, br. s, NH); $\delta_{\mathrm{C}}\left(100 \mathrm{MHz} ; \mathrm{CDCl}_{3}\right) 18.86\left(\mathrm{CH}_{3} \mathrm{CH}\right), 21.43\left(\mathrm{CH}_{3} \mathrm{CO}\right)$, $27.32\left(\mathrm{CH}_{3} \mathrm{~N}\right), 28.57\left(\mathrm{Me}_{3} \mathrm{C}\right), 47.93\left(\mathrm{CHCH}_{3}\right), 79.55\left(\mathrm{CMe}_{3}\right), 141.97(\mathrm{OCHN}), 155.32(\mathrm{C}=\mathrm{N})$ and $172.60(\mathrm{C}=\mathrm{O}) ; \mathrm{m} / \mathrm{z}(\mathrm{EI}) 244\left(\mathrm{MH}^{+}, 75 \%\right), 204,201,144,128,99(100), 59,57,43$ and 41.

(S)-5-(1-Dibenzylaminoethyl)-2,4-dimethyl-4,5-dihydro-1,3,4-oxadiazole $\quad(27) . \quad(S)-N, N$ Dibenzylalaninal 26 (0.12 g, $0.47 \mathrm{mmol}), N^{1}$-acetyl- $N^{2}$-methylhydrazine 9 (0.05 g, $\left.0.56 \mathrm{mmol}\right)$ and methyl propenoate $(0.33 \mathrm{~g}, 3.89 \mathrm{mmol})$ were dissolved in toluene $(50 \mathrm{~mL})$ and heated at reflux in the presence of $4 \AA$ molecular sieves for $16 \mathrm{~h}$. Removal of the solvent under reduced pressure and column chromatography of the residue (hexane: ethyl acetate 1:1 v/v) afforded the title compound 27 , a yellow oil $(0.01 \mathrm{~g}, 7 \%)$ as the only product isolated pure, although incompletely characterized; $\delta_{\mathrm{H}}\left(400 \mathrm{MHz} ; \mathrm{CDCl}_{3}\right) 1.29\left(3 \mathrm{H}, \mathrm{d}, J 7.4 \mathrm{~Hz}, \mathrm{CH}_{3} \mathrm{CH}\right), 2.30(3 \mathrm{H}, \mathrm{s}$, $\left.\mathrm{CH}_{3} \mathrm{C}=\mathrm{N}\right), 3.07\left(3 \mathrm{H}, \mathrm{s}, \mathrm{CH}_{3} \mathrm{~N}\right), 3.63\left(2 \mathrm{H}, \mathrm{d}, \mathrm{J} 10.3 \mathrm{~Hz}, \mathrm{C}_{6} \mathrm{H}_{5} \mathrm{CHH}\right), 3.67\left(1 \mathrm{H}, \mathrm{m}, \mathrm{CHCH}_{3}\right), 3.71$ $\left(2 \mathrm{H}, \mathrm{d}, J 10.3 \mathrm{~Hz}, \mathrm{C}_{6} \mathrm{H}_{5} \mathrm{CH} H\right), 6.94(1 \mathrm{H}, \mathrm{d}, J 5.0 \mathrm{~Hz}, \mathrm{NCHO})$ and 7.22-7.40 (10H, m, Ar-H). 
2-Benzyloxypropanal (28). Ethyl lactate $(7.40 \mathrm{~g}, 0.063 \mathrm{~mol})$ in dry THF $(20 \mathrm{~mL})$ was added dropwise over $1 \mathrm{~h}$ to sodium hydride ( $5.00 \mathrm{~g}$ of a $60 \%$ suspension in oil, $0.125 \mathrm{~mol}$ ) suspended in dry THF (120 mL). After stirring the mixture for $2 \mathrm{~h}$, benzyl bromide (21.27 g, $0.126 \mathrm{~mol})$ was added and the mixture stirred for a further $48 \mathrm{~h}$, before dropwise addition of methanol: diethyl ether $(1: 1 \mathrm{v} / \mathrm{v})$ to destroy excess sodium hydride. Saturated ammonium chloride solution was added and the resulting precipitate was filtered and washed with diethyl ether. The combined organic extracts were washed with saturated brine, dried $\left(\mathrm{MgSO}_{4}\right)$ and purified by column chromatography (hexane: ether 9:1 v/v) to afford methyl 2-benzyloxypropanoate $(4.31 \mathrm{~g}, 36 \%)$ that was used directly. To the foregoing ester $(1.98 \mathrm{~g}, 10.19 \mathrm{mmol})$ stirred in anhydrous toluene $(40 \mathrm{~mL})$ at $-50^{\circ} \mathrm{C}$ was added dropwise DIBAL $(10.16 \mathrm{~mL}$ of a $1.2 \mathrm{M}$ solution in toluene, 12.40 $\mathrm{mmol})$ and the solution stirred for $2.5 \mathrm{~h}$. A saturated solution of sodium metabisulfite $(60 \mathrm{~mL})$ was added and the mixture allowed to warm to $20^{\circ} \mathrm{C}$. The layers were separated and the organic phase was further extracted with saturated sodium metabisulfite solution $(3 \times 80 \mathrm{~mL})$. The combined aqueous layers were washed with diethyl ether $(3 \times 60 \mathrm{~mL})$ then cooled to $0^{\circ} \mathrm{C}$, the $\mathrm{pH}$ was raised to 11 with sodium hydroxide solution $(8 M)$ and the solution extracted with diethyl ether $(3 \times 50 \mathrm{~mL})$, washed with saturated brine $(75 \mathrm{~mL})$, dried $\left(\mathrm{MgSO}_{4}\right)$ and the solvent removed under reduced pressure. Purification by column chromatography (hexane: ethyl acetate 9:1 v/v) gave the title compound $\mathbf{2 8}$ as a colorless oil $(0.41 \mathrm{~g}, 25 \%)$; Found: $\mathrm{MNH}_{4}{ }^{+}, 182.1183 . \mathrm{C}_{10} \mathrm{H}_{12} \mathrm{O}_{2}$ requires $\mathrm{MNH}_{4}, 182.1181$; $v_{\max }$ (neat) $/ \mathrm{cm}^{-1} 3465,3032,2982,2869,2361,1736,1497,1455$, 1375 and 1094; $\delta_{\mathrm{H}}\left(300 \mathrm{MHz} ; \mathrm{CDCl}_{3}\right) 1.31\left(3 \mathrm{H}, \mathrm{d}, J 7.0 \mathrm{~Hz}, \mathrm{CH}_{3}\right), 3.87(1 \mathrm{H}, \mathrm{dq}, J 1.7$ and 7.0 $\left.\mathrm{Hz}, \mathrm{CHCH}_{3}\right), 4.57$ and 4.64 (each $\left.1 \mathrm{H}, \mathrm{d}, J 11.7 \mathrm{~Hz}, \mathrm{CH}_{2}\right), 7.28-7.36(5 \mathrm{H}, \mathrm{m}, \mathrm{Ar}-\mathrm{H})$ and $9.65(1 \mathrm{H}$, d, J 1.7 Hz, CHO); $\delta_{\mathrm{C}}\left(75 \mathrm{MHz} ; \mathrm{CDCl}_{3}\right) 15.26\left(\mathrm{CH}_{3}\right), 71.96\left(\mathrm{CH}_{2}\right), 79.41\left(\mathrm{CHCH}_{3}\right), 127.91$, 128.04 and 128.54 (Ar-CH), 137.37 (Ar-C) and 203.32 (CHO); $m / z$ (CI) $182\left(\mathrm{MNH}_{4}^{+}, 100 \%\right)$, $125,108,106,91$ and 61.

5-(1-Benzyloxyethyl)-2,4-dimethyl-4,5-dihydro-1,3,4-oxadiazole (29). The procedure described for the synthesis of 1-acetyl-4-methoxycarbonyl-2-methyl-3-phenyltetrahydropyrazoles 10 and 11 was followed, but using 2-benzyloxypropanal $28(0.37 \mathrm{~g}, 2.25 \mathrm{mmol}), N^{1}$ acetyl- $N^{2}$-methylhydrazine $9(0.20 \mathrm{~g}, 2.27 \mathrm{mmol})$, methyl propenoate $(0.40 \mathrm{~g}, 4.66 \mathrm{mmol})$ and toluene $(25 \mathrm{~mL})$, and heating at reflux for $24 \mathrm{~h}$. Column chromatography (hexane: ethyl acetate $1: 1 \mathrm{v} / \mathrm{v})$ afforded the title compound 29 as a yellow oil $(0.06 \mathrm{~g}, 11 \%)$ as the only product isolated pure; $\delta_{\mathrm{H}}\left(300 \mathrm{MHz} ; \mathrm{CDCl}_{3}\right) 1.40\left(3 \mathrm{H}, \mathrm{d}, J 6.4 \mathrm{~Hz}, \mathrm{CH}_{3} \mathrm{CH}\right), 2.32\left(3 \mathrm{H}, \mathrm{s}, \mathrm{CH}_{3} \mathrm{C}=\mathrm{N}\right), 3.13(3 \mathrm{H}, \mathrm{s}$, $\left.\mathrm{CH}_{3} \mathrm{~N}\right), 4.18\left(1 \mathrm{H}\right.$, quintet, $\left.J 6.4 \mathrm{~Hz}, \mathrm{CHCH}_{3}\right), 4.55\left(2 \mathrm{H}, \mathrm{s}, \mathrm{C}_{6} \mathrm{H}_{5} \mathrm{CH}_{2}\right), 6.84(1 \mathrm{H}, \mathrm{d}, J 6.4 \mathrm{~Hz}$, $\mathrm{NCHO})$ and 7.19-7.37 (5H, m, Ar-H).

1-Acetyl-2-benzyl-4-methoxycarbonyl-3-(2-propyl)tetrahydropyrazole (31). $N^{1}$-Acetyl- $N^{2}$ benzylhydrazine 30 (0.32 g, $1.95 \mathrm{mmol})$, 2-methylpropanal (0.15 g, $2.09 \mathrm{mmol})$, methyl propenoate $(0.52 \mathrm{~g}, 6.00 \mathrm{mmol})$, toluene $(10 \mathrm{~mL})$ and $4 \AA$ molecular sieves were placed in a glass tube, which was then sealed with a screw-cap. The mixture was heated for $72 \mathrm{~h}$, after which the solvent was removed under reduced pressure. Column chromatography (hexane: ethyl acetate 2:1 $\mathrm{v} / \mathrm{v})$ of the residue gave the title compound $31(0.03 \mathrm{~g}, 4 \%)$ as a yellow oil; Found: $\mathrm{MH}^{+}$, 305.1863. $\mathrm{C}_{17} \mathrm{H}_{24} \mathrm{~N}_{2} \mathrm{O}_{3}$ requires $\mathrm{MH}, 305.1865 ; v_{\max }\left(\mathrm{CHCl}_{3}\right) / \mathrm{cm}^{-1} 3021,2963,1733,1641$, 
1437, 1369, 1216 and 700; $\delta_{\mathrm{H}}\left(300 \mathrm{MHz}, \mathrm{CDCl}_{3}\right) 0.83\left(3 \mathrm{H}, \mathrm{d}, J 6.9 \mathrm{~Hz}, \mathrm{CH} \mathrm{CH}_{3}\right), 0.85(3 \mathrm{H}, \mathrm{d}, J$ $\left.7.1 \mathrm{~Hz}, \mathrm{CH}_{3} \mathrm{CH}\right), 1.39$ (1H, m, CHMe 2$), 1.92$ (3H, s, $\left.\mathrm{CH}_{3} \mathrm{CON}\right), 3.06-3.12$ (2H, m, $\mathrm{CHCHMe}_{2}$ and $\left.\mathrm{CHCO}_{2}\right), 3.75(1 \mathrm{H}, \mathrm{m}, 5-\mathrm{CHH}), 3.79\left(3 \mathrm{H}, \mathrm{s}, \mathrm{CO}_{2} \mathrm{CH}_{3}\right), 3.83\left(1 \mathrm{H}, \mathrm{d}, J 11.9 \mathrm{~Hz}, \mathrm{C}_{6} \mathrm{H}_{5} \mathrm{CH}\right)$, $3.90\left(1 \mathrm{H}, \mathrm{d}, J 11.9 \mathrm{~Hz}, \mathrm{C}_{6} \mathrm{H}_{5} \mathrm{CH} H\right), 4.52(1 \mathrm{H}, \mathrm{dd}, J 9.8,12 \mathrm{~Hz}, 5-\mathrm{CH})$ and 7.26-7.38 (5H, m, $\mathrm{Ar}-\mathrm{H}) ; \delta_{\mathrm{C}}\left(75 \mathrm{MHz}, \mathrm{CDCl}_{3}\right) 18.99\left(\mathrm{CH}_{3} \mathrm{CH}\right), 20.09\left(\mathrm{CH}_{3} \mathrm{CH}\right), 20.72\left(\mathrm{CH}_{3} \mathrm{CON}\right), 32.51$ $\left(\mathrm{CHMe}_{2}\right), 44.65\left(5-\mathrm{CH}_{2}\right), 47.56 \quad\left(\mathrm{CHCO}_{2}\right), \quad 52.54 \quad\left(\mathrm{CO}_{2} \mathrm{CH}_{3}\right), \quad 61.84 \quad\left(\mathrm{C}_{6} \mathrm{H}_{5} \mathrm{CH}_{2}\right), \quad 74.86$ $\left(\mathrm{CHCHMe}_{2}\right), 127.93,128.45$ and 130.29 (Ar-CH), $136.29(\mathrm{Ar}-\mathrm{C}), 171.59$ and $174.37(\mathrm{C}=\mathrm{O}) ; \mathrm{m} / z$ (EI) $305\left(\mathrm{MH}^{+}, 2 \%\right), 261,171,111,91(100), 69,65$ and 43.

1-tert-Butoxycarbonyl-4-methoxycarbonyl-2-methyl-3-phenyltetrahydropyrazole (33). The procedure described for the synthesis of 1-acetyl-4-methoxycarbonyl-2-methyl-3-phenyltetrahydropyrazoles 10 and 11 was followed, but using benzaldehyde $(0.26 \mathrm{~g}, 2.46 \mathrm{mmol}), N^{1}$ tert-butoxycarbonyl- $N^{2}$-methylhydrazine $32(0.35 \mathrm{~g}, 2.39 \mathrm{mmol})$, methyl propenoate $(0.42 \mathrm{~g}$, $4.89 \mathrm{mmol})$ and toluene $(25 \mathrm{~mL})$. Column chromatography (hexane: ethyl acetate 2:1 v/v) afforded the title compound $33(0.14 \mathrm{~g}, 18 \%)$ as a colorless oil; Found: $\mathrm{M}^{+}, 320.1735$. $\mathrm{C}_{17} \mathrm{H}_{24} \mathrm{~N}_{2} \mathrm{O}_{4}$ requires $\mathrm{M}, 320.1736 ; v_{\max }\left(\mathrm{CHCl}_{3}\right) / \mathrm{cm}^{-1}$ 2982, 1739, 1710, 1390, 1369, 1312, 1169 and 1121; $\delta_{\mathrm{H}}\left(300 \mathrm{MHz}, \mathrm{CDCl}_{3}\right) 1.54\left(3 \mathrm{H}, \mathrm{s}, \mathrm{Me}_{3} \mathrm{C}\right), 2.72\left(3 \mathrm{H}, \mathrm{s}, \mathrm{CH}_{3} \mathrm{~N}\right), 3.42\left(3 \mathrm{H}, \mathrm{s}, \mathrm{CO}_{2} \mathrm{CH}_{3}\right)$, $3.72\left(2 \mathrm{H}, \mathrm{m}, \mathrm{CHCO}_{2}, 5-\mathrm{CHH}\right), 4.00(1 \mathrm{H}, \mathrm{dd}, J 10.8,14.3 \mathrm{~Hz}, 5-\mathrm{CH} H), 4.32(1 \mathrm{H}, \mathrm{d}, J 6.4 \mathrm{~Hz}$, $\left.\mathrm{C}_{6} \mathrm{H}_{5} \mathrm{CH}\right)$ and 7.17-7.30 (5H, m, Ar-H); $\delta_{\mathrm{C}}\left(75 \mathrm{MHz}, \mathrm{CDCl}_{3}\right) 28.52\left(\mathrm{Me}_{3} \mathrm{C}\right), 45.54\left(\mathrm{CH}_{3} \mathrm{~N}\right), 46.36$ $\left(5-\mathrm{CH}_{2}\right), 48.33\left(\mathrm{CHCO}_{2}\right), 51.65\left(\mathrm{CO}_{2} \mathrm{CH}_{3}\right), 71.61\left(\mathrm{C}_{6} \mathrm{H}_{5} \mathrm{CH}\right), 80.39\left(\mathrm{CMe}_{3}\right), 126.70,127.69$ and 128.27 (Ar-CH), 137.85 (Ar-C), $154.79(\mathrm{CON})$ and $170.19\left(\mathrm{CO}_{2} \mathrm{CH}_{3}\right) ; m / z(\mathrm{EI}) 320\left(\mathrm{M}^{+}, 3 \%\right)$, $265,219,187,159,133,115,91,77,57(100)$ and 41.

\section{Acknowledgements}

We wish to acknowledge the Open University for a studentship (S. J. H), the EPSRC National Mass Spectrometry Service Centre (University of Wales Swansea) for some mass spectral data, and the EPSRC X-Ray Crystallography Service (University of Southampton) for crystal structure determinations.

\section{References and Footnotes}

1. (a) Jones, R. C. F.; Hollis S. J.; Iley, J. N. Tetrahedron: Asymmetry, 2000, 11, 3273. (b) Jones, R. C. F.; Gilbert, I. H.; Rees D. C.; Crockett, A. K. Tetrahedron 1995, 51, 6315.

2. $\quad c f:$ Dutta, A. S.; Morley, J. S. J. Chem. Soc., Perkin Trans. 1 1975, 1712. Mish, M. R.; Guerra, F. M.; Carreira, E. M. J. Am. Chem. Soc. 1997, 119, 8379.

3. See, for example: Advances in Amino Acid Mimetics and Peptidomimetics, Abell, A., Ed.; Jai Press Inc, Stamford (USA), 1999; Vol. 2. 
4. Grashey, R. in 1,3-Dipolar Cycloaddition Chemistry; Padwa, A., Ed.; John Wiley: New York, 1984, p. 733.

5. See, for example: Sun, B.; Adachi, K.; Noguchi, M. Tetrahedron 1996 52, 901. Jacobi, P. A.; Martinelli, M. J.; Polanc, S. J. Am. Chem. Soc. 1984, 106, 5594. Oppolzer, W. Tetrahedron Lett. 1970, 3091.

6. Oppolzer, W. Tetrahedron Lett. 1970, 2199.

7. Huisgen, R.; Grashey, R.; Sauer, J. The Chemistry of Alkenes, Patai, S., Ed.; WileyInterscience, New York, 1964; p. 849 et seq.

8. Roussi, F.; Chauveau, A.; Bonin, M.; Micouin, L.; Husson, H.-P. Synthesis 2000, 1170.

9. Chauveau, A.; Martens, T.; Bonin, M.; Micouin, L.; Husson, H.-P. Synthesis 2002, 1885.

10. Condon, F. E. J. Org. Chem. 1972, 37, 3608.

11. Experiments carried out in an NMR tube with $d_{8}$-toluene showed that heating for $72 \mathrm{~h}$ was required for complete reaction of the dipole to occur.

12. Dorn, H. Tetrahedron Lett. 1985, 26, 5123.

13. Kanemasa, S.; Tomoshige, N.; Wada, E.; Tsuge, O. Bull. Chem. Soc. Jpn. 1989, 62, 3944.

14. In all cycloadditions carried out with mono-substituted dipolarophiles, where two 4substituted pyrazolidines were observed, the isomer identified as syn-was less polar in TLC analysis on silica than the anti-isomer. The chemical shift of the methine proton at C-4 was also found to be greater for the $s y n$-isomer than for the anti-isomer $(\delta 3.94 \mathrm{ppm}$ for $\mathbf{1 0}$ and $\delta$ 3.45 for 11). Values for this proton in 21 and 22 are $\delta 3.76$ and $\delta 3.05$, respectively.

15. Chung, Y. J.; Ryu, E. J.; Keum, G.; Kim, B. H. Bioorg. Med. Chem. 1996, 4, 209.

16. Reetz, M. T.; Drewes, M. W.; Schmitz, A. Angew. Chem., Int. Ed. Engl. 1987, 26, 1141.

17. Calabretta, R.; Gallina, C.; Giordano, C. Synthesis 1991, 536.

18. Lenman, M. M.; Lewis, A.; Gani, D. J. Chem. Soc., Perkin Trans. 1 1997, 2297.

19. See: Perrin, D. D.; Armarego, W. L. F. Purification of Laboratory Chemicals, $3^{\text {rd }}$ Edn.; Pergamon Press: Oxford, 1988. 